\title{
Article
}

\section{Regional variation in acute stroke care organisation}

Muñoz Venturelli, Paula, Robinson, Thompson, Lavados, Pablo M., Olavarría, Verónica V., Arima, Hisatomi, Billot, Laurent, Hackett, Maree, Lim, Joyce Y., Middleton, Sandy, Pontes-Neto, Octavio, Peng, Bin, Cui, Liying, Song, Lily, Mead, Gillian, Watkins, Caroline Leigh, Lin, Ruey-Tay, Lee, Tsong-Hai, Pandian, Jeyaraj, de Silva, H. Asita and Anderson, Craig S.

Available at http://clok.uclan.ac.uk/16125/

Muñoz Venturelli, Paula, Robinson, Thompson, Lavados, Pablo M., Olavarría, Verónica V., Arima, Hisatomi, Billot, Laurent, Hackett, Maree ORCID: 00000003-1211-9087, Lim, Joyce Y., Middleton, Sandy et al (2016) Regional variation in acute stroke care organisation. Journal of the Neurological Sciences, 371 . pp. 126-130. ISSN 0022510X

It is advisable to refer to the publisher's version if you intend to cite from the work. http://dx.doi.org/10.1016/j.jns.2016.10.026

For more information about UCLan's research in this area go to http://www.uclan.ac.uk/researchgroups/ and search for <name of research Group>.

For information about Research generally at UCLan please go to http://www.uclan.ac.uk/research/

All outputs in CLoK are protected by Intellectual Property Rights law, including Copyright law. Copyright, IPR and Moral Rights for the works on this site are retained by the individual authors and/or other copyright owners. Terms and conditions for use of this material are defined in the policies page.

\section{CLoK}

Central Lancashire online Knowledge www.clok.uclan.ac.uk

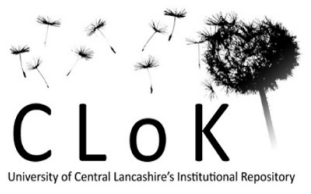




\section{REGIONAL VARIATION IN ACUTE STROKE CARE ORGANISATION}

Paula Muñoz-Venturelli, ${ }^{1,2}$ Thompson Robinson, ${ }^{3}$ Pablo M. Lavados, ${ }^{2,4}$ Verónica V.

Olavarría, ${ }^{2}$ Hisatomi Arima, ${ }^{5}$ Laurent Billot, ${ }^{1}$ Maree L. Hackett, ${ }^{1,6}$ Joyce Y. Lim, ${ }^{1}$ Sandy Middleton, ${ }^{7}$ Octavio Pontes-Neto, ${ }^{8}$ Bin Peng, ${ }^{9}$ Liying Cui, ${ }^{9}$ Lily Song, ${ }^{10}$ Gillian Mead, ${ }^{11}$ Caroline Watkins, ${ }^{6,7}$ Ruey-Tay Lin, ${ }^{12}$ Tsong-Hai Lee, ${ }^{13}$ Jeyaraj Pandian, ${ }^{14}$ H. Asita de Silva, ${ }^{15}$ Craig S. Anderson, ${ }^{1,16}$ for the HeadPoST Investigators

${ }^{1}$ The George Institute for Global Health, University of Sydney, Sydney, Australia ${ }^{2}$ Unidad de Neurología Vascular, Servicio de Neurología, Departamento de Medicina, Clínica Alemana de Santiago, Facultad de Medicina Clínica Alemana Universidad del Desarrollo, Santiago, Chile

${ }^{3}$ Department of Cardiovascular Sciences and NIHR Biomedical Research Unit for Cardiovascular Disease, University of Leicester, Leicester, UK

${ }^{4}$ Departamento de Ciencias Neurológicas, Facultad de Medicina, Universidad de Chile, Santiago, Chile.

${ }^{5}$ Department of Preventive Medicine and Public Health, Faculty of Medicine, Fukuoka University, Fukuoka, Japan

${ }^{6}$ College of Health and Wellbeing, University of Central Lancashire, Preston, UK

${ }^{7}$ Nursing Research Institute, St Vincents Health Australia (Sydney) and Australian Catholic University, Australia

${ }^{8}$ Stroke Service, Neurology Division, Ribeirão Preto School of Medicine, University of São Paulo, Ribeirão Preto, Brazil 
${ }^{9}$ Department of Neurology, Peking Union Medical College Hospital, Beijing, China

${ }^{10}$ Department of Neurology, Shanghai 85th Hospital of PLA, Shanghai, China

${ }^{11}$ Department of Geriatric Medicine, Centre for Clinical Brain Sciences, University of Edinburgh, Scotland, UK

${ }^{12}$ Department of Neurology, Kaohsiung Medical University Chung-Ho Memorial

Hospital, Taiwan

${ }^{13}$ Department of Neurology, Linkou Chang Gung Memorial Hospital and College of

Medicine, Chang Gung University, Taoyuan, Taiwan

${ }^{14}$ Department of Neurology, Christian Medical College, Ludhiana, Punjab, India

${ }^{15}$ Clinical Trials Unit, Department of Pharmacology, Faculty of Medicine, University of

Kelaniya, Ragama, Sri Lanka

${ }^{16}$ Neurology Department, Royal Prince Alfred Hospital, Sydney, Australia

\section{Author for correspondence:}

Professor Craig S. Anderson

The George Institute for Global Health

PO Box M201, Missenden Road, NSW 2050, AUSTRALIA

T: +61-2-9993-4500, Email: canderson@georgeinstitute.org.au

Cover title: Stroke care organisation

Key words: stroke, stroke care, acute ischemic stroke, intracerebral hemorrhage,

thrombolysis, hospital organisation.

Word count: 3864

\section{Itemized list of tables and figures:}

Tables: 
Table 1: Hospital characteristics by country income

Table 2: Protocols, discharge plans, rehabilitation, follow-up services and continuing education by country income

Table 3: Human resources in stroke care by country income

\section{Figures:}

Figure: Percentage of hospitals having different health professionals involved in the management of stroke patients, by country income

Any underlying research material will be made available upon a formal request process through the Research Office of The George Institute Australia in Sydney. 


\section{Abstract (227 words)}

Background: Few studies have assessed regional variation in the organisation of stroke services, particularly health care resourcing, presence of protocols and discharge planning.

Aim: To compare stroke care organisation within middle- (MIC) and high-income country (HIC) hospitals participating in the Head Positioning in Stroke Trial (HeadPoST).

Methods: HeadPoST is an on-going international multicenter crossover clusterrandomized trial of 'sitting-up' versus 'lying-flat' head positioning in acute stroke. As part of the start-up phase, all hospitals were required to complete a stroke care organisation questionnaire.

Results: 94 hospitals from 9 countries completed the questionnaire, 51 corresponding to MIC and 43 to HIC. Most participating hospitals had a dedicated stroke care unit/ward, with access to diagnostic services and expert stroke physicians, and offering intravenous thrombolysis. There was a no difference for the presence of a dedicated multidisciplinary stroke team, although greater access to a broad spectrum of rehabilitation therapists in HIC compared to MIC hospitals was observed. Significantly more patients arrived within a 4-hour window of symptoms onset in HIC hospitals (41 vs. $13 \%$; $\mathrm{P}<0.001$ ), and a significantly higher proportion of acute ischemic stroke patients received intravenous thrombolysis ( 10 vs. $5 \%$; $\mathrm{P}=0.002$ ) compared to MIC hospitals.

Conclusions: Although all hospitals provided advanced care for people with stroke, differences were found in stroke care organisation and treatment. Future multilevel 
analyses aim to determine the influence of specific organisational factors on patient outcomes. 


\section{Introduction}

Organisation of stroke care is a key factor in patient outcomes, having been shown to significantly reduce mortality and disability.(1) Presence of local protocols and pathways, adequate health care resources (including staff), regular quality assessments, and discharge planning appear to positively impact care. However, wide variations have been reported in acute stroke care processes, costs and outcomes worldwide.(2, 3) In addition, there are fewer stroke units in lower-income countries, where acute care tends to be inconsistent, with large differences in average length of hospital stay between countries.(3)

Disparity in stroke care is important as stroke disproportionately affects people in lowand middle-income countries (MIC), compared to high-income countries (HIC).(4) This has resulted in the World Stroke Organisation assigning the highest priority to the detection of barriers and implementation of evidence-based interventions for stroke care.(5) Unfortunately, gaps in information about stroke organisation care from different sites, and in particular according to country income, have hampered the implementation of specifically designed and focussed interventions.

\footnotetext{
Aim

To describe the organisation of care in stroke units from different countries participating in the Head Positioning in Stroke Trial (HeadPoST), and to compare health care resourcing, presence of protocols and discharge planning by country income.
} 


\section{Methods}

HeadPoST is an international, cluster-randomised, crossover, open, blinded outcomeassessed clinical trial to determine the effects of lying-flat $\left(0^{\circ}\right)$ compared with sitting-up $\left(\geq 30^{\circ}\right)$ head positioning in the first 24 hours of hospital admission for patients with acute stroke. Methods have been published in detail elsewhere.(6) Hospitals with established acute stroke care programs have started recruitment and are at different stages of the trial. As part of the start-up phase, principal investigators at each hospital were required to complete a previously published Hospital Organisation Questionnaire (HOQ) designed by the study investigators to determine how stroke care is organized.(6) HOQ has only closed questions on a wide variety of stroke topics, including hospital details, stroke pathways, emergency department characteristics, imaging and clinical resources, acute treatment strategies, stroke care and stroke unit, discharge plans, rehabilitation and follow-up services, continuing education, research and quality improvement. Answers were uploaded into an electronic database, identified only by the study assigned number. The HOQ was designed in English and was also translated into Chinese.

To be included in HeadPoST, hospitals were required to have an established acute stroke care program within a geographically defined area and a sufficient projected throughput of patients to ensure feasibility of recruitment within a short time frame. Local Ethics and regulatory entities have approved the study in the different countries. Study data were collected and managed using REDCap electronic data capture tools hosted at The George Institute for Global Health.(7)

\section{Statistical analysis}

Chi squared or Fisher tests were used to compare categorical variables, and Mann- 
Whitney test was used for continuous variables. IBM SPSS Statistics version 21 was used. A two-sided $\mathrm{P}$ value $<0.05$ was considered significant.

\section{Results}

Ninety-four hospitals completed the HOQ between 26th August 2014 and 1st February 2016. According to current world data bank criteria,(8) hospitals were allocated in the MIC (51 hospitals; India (5), Sri Lanka (4), Brazil (1), Colombia (1), China (40)), or HIC groups (43; Australia (6), Chile (6), UK (28), Taiwan (3)).

Health service capacity for stroke care

Hospital characteristics are depicted in Table 1, with most having a dedicated stroke care unit/ward. MIC hospitals were more likely to be academic, with a higher number of beds, including dedicated stroke beds, and also more likely to be located in a metropolitan area (Table 1). Access to diagnostic and other support services was comparable; with local urgent CT available in 49 [98\%] of MIC and 43 [100\%] of HIC hospitals, and emergency laboratory facilities in 49 [98\%] and 43 [100\%] of MIC and HIC hospitals, respectively. Most hospitals had access to on-going rehabilitation at the admitting or another hospital, and offered routine outpatient clinic follow-up review for all stroke patients (Table 2).

Stroke pathway for intravenous thrombolysis

Compared to MIC hospitals, HIC hospitals had more stroke patients arriving by ambulance and within 4 hours of symptom onset; 81\% (interquartile range [IQR] 75-90) vs. $21 \%$ (IQR 10-48), $\mathrm{P}<0.001$, and $35 \%$ (IQR 25-55) vs. $10 \%$ (IQR 6-20), $\mathrm{P}<0.001$. In addition, HIC hospitals received more frequently a pre-alert from the ambulance service 
to the Emergency Department (ED) prior to patient arrival (39 [91\%] vs. 36 [72\%], $\mathrm{P}=0.034$ ), and have an acute "code stroke" pager system for potential thrombolysis patients with medical staff from the stroke service available in ED (38 [88\%] vs. 21 [42\%] hospitals, $\mathrm{P}<0.001)$. Though there was no significant difference in the proportion of HIC and MIC hospitals offering thrombolysis (Table 1), more acute ischemic stroke (AIS) patients received rtPA in HIC compared to MIC hospitals (10\% [IQR 7-15] vs. 5\% [IQR 1-10], respectively, $\mathrm{P}=0.002)$. Interventional neuroradiology and neurosurgery were more likely to be available in MIC hospitals (Table 1).

\section{Human resources in stroke care}

All hospitals had access to physicians with stroke expertise and a dedicated multidisciplinary team. MIC hospitals had a higher total number, as well as range, of medical officers, but this difference is associated with a higher number of dedicated stroke beds in MIC hospitals. No difference was detected in the number of patients to one nurse or health assistant in ED or stroke care unit between MIC and HIC hospitals. However, HIC hospitals offer access to a broader range of rehabilitation therapists compared to MIC. Further details of staffing are provided in Table 3 and Figure.

Protocols, discharge plans and quality assurance

The availability of a special pathway, or ward for stroke care was non-significantly higher in HIC hospitals (41 [95\%] vs. 40 [82\%], $\mathrm{P}=0.056$ ), as were referral protocols to different health professionals more common (Table 2). However, most hospitals had local protocols available for fever control, blood glucose management and swallow assessment, with no significant difference between HIC and MIC hospitals (Table 2). In addition, access to on-going rehabilitation, discharge services with home-based 
rehabilitation and assessment, and routine outpatient follow-up review was similar between HIC and MIC hospitals, though home nursing service and palliative care were more frequently available in HIC hospitals (Table 2). Continuing stroke management education was available in most hospitals, though quality improvement activity was more frequent in HIC hospitals (Table 2).

\section{Discussion}

Most HeadPoST-participating hospitals had advanced health service capacity for stroke care following the recently published World Stroke Organisation classification,(5) with access to coordinated stroke care, physicians with stroke expertise, advanced diagnostic and interventional services, rehabilitation and discharge programs. We acknowledge that this manuscript relates to selected hospitals participating in a clinical trial, which may not necessarily represent other institutions in their country, and that China and the United Kingdom account for the majority of hospitals. Nonetheless, this article provides description of stroke care in MIC hospitals, which are currently under-represented in literature with most publications referring to Europe,(2) United States,(9) Canada(10) and Taiwan.(11) There is therefore an important opportunity to discuss stroke care between MIC and HIC health systems, and in particular the wide array of stroke care in different hospitals from a number of countries, with particular emphasis on facilities, patient pathways, staffing, and protocols and quality improvement activities.

Though most MIC hospitals have implemented pre-hospital ambulance pathways for the timely arrival of patients, have an emergency call number and are located in a metropolitan area, our data show that most stroke patients do not use the ambulance 
service to go to hospital and therefore fail to arrive within the time window for intravenous thrombolysis. Importantly, responses to the HOQ indicate that service organisation in MIC hospitals is available to ensure appropriate door-to-needle time in those patients arriving within a thrombolysis time window. These data stress the need for comprehensive strategies to provide successful pre-hospital stroke care, with networks ensuring rapid access to health care, timely medical evaluation and diagnosis, and tailored treatment. In addition, educational programs and campaigns to raise awareness of stroke symptoms and the importance of immediate consultation can aid in improving the number of patients being evaluated during the reperfusion window and thus increase the number of rtPA-treated patients in MIC.(12, 13)

In contrast to thrombolysis management of ischemic stroke, MIC hospitals had more evidence of stroke care focused on intracerebral haemorrhage (ICH) management, including local clinical pathways and on-site neurosurgical availability. This probably reflects that most MIC hospitals in our study come from Asian regions, where ICH is highly prevalent,(14) but does confirm that it should be possible to implement care pathways for ischemic stroke too. This is important, because stroke distribution in Asian regions is rapidly changing with industrialization, causing an increase in ischemic stroke, a reduction in $\mathrm{ICH}$, and a need to provide pathways and protocols similar to those reported in western countries.(15)

MIC hospitals were predominantly larger with higher patient-flow, and this 'volumeoutcome relationship' has been shown to be an important predictor of short-term mortality in some,(16) though not all,(17) stroke studies. However, participating HIC hospitals may have adopted a two-tiered (or 'hub-and-spoke') model of stroke care in 
which a central 'comprehensive stroke centre' serves a number of smaller hospitals working as 'primary stroke centres' for thrombolysis of AIS patients, using protocols for transfer to larger hospitals for interventional therapies and telemedicine assistance.(18) Therefore, the HOQ may not have captured the entire range of treatment options available to patients in participating HIC hospitals.

With respect to the rehabilitation component of the stroke pathway, MIC hospitals had fewer multidisciplinary stroke care and referral protocols to different health professionals when compared to HIC hospitals. This may have an adverse impact on stroke outcome, as the presence of occupational therapy or physiotherapy, stroke team assessment and continuing education are key for stroke care, showing an effect that is independent of the severity of the disease. $(9,10)$ Indeed, escalating levels of organised care, measured by the Organised Care Index - a summary score based on hospital availability of occupational therapy or physiotherapy, stroke team assessment of patients, and admission to a stroke unit - were independent predictors of improved stroke survival in Canada(10) and Europe.(2) How these differences in stroke care impact on patient outcome is not known.

In conclusion, differences exist in stroke care organisation within hospitals with advanced health service capacity that have been selected to participate in a large-scale stroke trial. Importantly, these differences exist along the whole stroke care pathway, from prehospital, including population awareness of stroke, through acute and rehabilitation hospital care, to long-term community care, including palliative care services. Whilst these differences may reflect variations in funding and/or national health programs between MIC and HIC hospitals, it is important to consider the potential impact on 
patient outcome, including in the context of the on-going HeadPoST study. Future multilevel analyses will be required to determine the influence of these organisational factors on patient outcome in different stroke subtypes within MIC and HIC health economies.

\section{Acknowledgements:}

Funding: The National Health and Research Medical Council (NHMRC) of Australia. During the completion of this work MLH was in receipt of a National Heart Foundation Future Leader Fellowship 100034.

Conflict of interest: TR has received consultancy payments from Bayer, Boehringer Ingelheim, Daiichi Sankyo, and his institution has received grant funding from the National Institute of Health Research, British Heart Foundation, Stroke Association, and the Engineering and Physical Sciences Research Council. PML has received research grant support Bayer, Boehringer Ingelheim and AstraZeneca and receives research support from CONICYT-FONIS, Clínica Alemana de Santiago and The George Institute for Global Health. He has received speaker honoraria from Bayer. VVO has received research grant support from Clínica Alemana de Santiago. CSA has received travel reimbursement and honorarium from Takeda China. Other authors have no conflicts of interest to declare. 


\section{References}

1. Schwamm LH, Pancioli A, Acker JE, 3rd, et al. Recommendations for the establishment of stroke systems of care: recommendations from the American Stroke Association's Task Force on the Development of Stroke Systems. Stroke. 2005;36(3):690-703.

2. Ayis SA, Coker B, Bhalla A, et al. Variations in acute stroke care and the impact of organised care on survival from a European perspective: the European Registers of Stroke (EROS) investigators. J Neurol Neurosurg Psychiatry. 2013;84(6):604-12.

3. Kaur P, Kwatra G, Kaur R, Pandian JD. Cost of stroke in low and middle income countries: a systematic review. Int J Stroke. 2014;9(6):678-82.

4. Feigin VL, Forouzanfar MH, Krishnamurthi R, et al. Global and regional burden of stroke during 1990-2010: findings from the Global Burden of Disease Study 2010. Lancet. 2014;383(9913):245-54.

5. Lindsay P, Furie KL, Davis SM, Donnan GA, Norrving B. World Stroke Organization global stroke services guidelines and action plan. Int J Stroke. 2014;9 Suppl A100:4-13.

6. Munoz-Venturelli P, Arima H, Lavados P, et al. Head Position in Stroke Trial (HeadPoST)--sitting-up vs lying-flat positioning of patients with acute stroke: study protocol for a cluster randomised controlled trial. Trials. 2015;16:256.

7. Harris PA, Taylor R, Thielke R, Payne J, Gonzalez N, Conde JG. Research electronic data capture (REDCap)--a metadata-driven methodology and workflow process for providing translational research informatics support. Journal of biomedical informatics. 2009;42(2):377-81. 
8. The World Bank. Country and Lending Groups 2016. Available from: http://data.worldbank.org/about/country-and-lending-groups.

9. Reeves MJ, Gargano J, Maier KS, et al. Patient-level and hospital-level determinants of the quality of acute stroke care: a multilevel modeling approach. Stroke. 2010;41(12):2924-31.

10. Saposnik G, Fang J, O'Donnell M, et al. Escalating levels of access to in-hospital care and stroke mortality. Stroke. 2008;39(9):2522-30.

11. Tung YC, Jeng JS, Chang GM, Chung KP. Processes and outcomes of ischemic stroke care: the influence of hospital level of care. International journal for quality in health care : journal of the International Society for Quality in Health Care / ISQua. 2015;27(4):260-6.

12. Chiti A, Fanucchi S, Sonnoli C, Barni S, Orlandi G. Stroke symptoms and the decision to call for an ambulance: turn on people's minds! Stroke. 2007;38(7):e58-9. 13. Mosley I, Nicol M, Donnan G, Patrick I, Dewey H. Stroke symptoms and the decision to call for an ambulance. Stroke. 2007;38(2):361-6.

14. Zhao D, Liu J, Wang W, et al. Epidemiological transition of stroke in China: twenty-one-year observational study from the Sino-MONICA-Beijing Project. Stroke. 2008;39(6):1668-74.

15. Mehndiratta MM, Khan M, Mehndiratta P, Wasay M. Stroke in Asia: geographical variations and temporal trends. J Neurol Neurosurg Psychiatry. 2014;85(12):1308-12.

16. Saposnik G, Baibergenova A, O'Donnell M, et al. Hospital volume and stroke outcome: does it matter? Neurology. 2007;69(11):1142-51. 
17. Heuschmann PU, Kolominsky-Rabas PL, Misselwitz B, et al. Predictors of inhospital mortality and attributable risks of death after ischemic stroke: the German Stroke Registers Study Group. Arch Intern Med. 2004;164(16):1761-8.

18. Jauch EC, Saver JL, Adams HP, Jr., et al. Guidelines for the early management of patients with acute ischemic stroke: a guideline for healthcare professionals from the American Heart Association/American Stroke Association. Stroke. 2013;44(3):870-947. 
Table 1: Hospital characteristics by country income

\begin{tabular}{|c|c|c|c|}
\hline & MIC & HIC & $P$ value \\
\hline Academic & 47 (94\%) & $29(67 \%)$ & 0.001 \\
\hline Number of beds & $\begin{array}{l}1000(800- \\
1800)\end{array}$ & $\begin{array}{l}600(404- \\
745)\end{array}$ & $<0.001$ \\
\hline Metropolitan & $43(84 \%)$ & $26(61 \%)$ & 0.011 \\
\hline Intensive care unit & $51(100 \%)$ & $43(100 \%)$ & NS \\
\hline Neurosurgery & 45 (90\%) & $16(37 \%)$ & $<0.001$ \\
\hline Interventional neuroradiology & $33(67 \%)$ & 15 (35\%) & 0.003 \\
\hline Dedicated stroke ward/unit & $42(84 \%)$ & $41(95 \%)$ & NS \\
\hline Number of dedicated stroke beds & $51(10-100)$ & $28(10-38)$ & 0.009 \\
\hline $\begin{array}{l}\text { Number of acute stroke patients admitted } \\
\text { annually }\end{array}$ & $\begin{array}{l}740(400- \\
2148)\end{array}$ & $\begin{array}{l}576(400- \\
818)\end{array}$ & 0.046 \\
\hline Intravenous thrombolysis (rtPA) & 46 (92\%) & $42(98 \%)$ & NS \\
\hline Stroke database & 34 (68\%) & $40(93 \%)$ & 0.004 \\
\hline
\end{tabular}


Table 2: Protocols, discharge plans, rehabilitation, follow-up services and continuing education by country income

\begin{tabular}{|c|c|c|c|}
\hline & MIC & HIC & $\mathrm{P}$ value \\
\hline $\begin{array}{l}\text { Special pathway, ward or service organisation } \\
\text { at the hospital for the management of patients } \\
\text { with acute stroke }\end{array}$ & $40(82 \%)$ & $41(95 \%)$ & NS \\
\hline $\begin{array}{l}\text { Current ED pathway/checklist/protocol for } \\
\text { AIS rapid triage and treatment }\end{array}$ & $48(94 \%)$ & $38(88 \%)$ & NS \\
\hline Clinical pathway/checklist for ICH patients & $42(86 \%)$ & $28(68 \%)$ & 0.048 \\
\hline $\begin{array}{l}\text { Standardised protocol for administering } \\
\text { intravenous rtPA }\end{array}$ & $45(100 \%)$ & $41(100 \%)$ & NS \\
\hline $\begin{array}{l}\text { Guidelines for acute treatment of stroke } \\
\text { patients in the stroke care unit }\end{array}$ & $44(94 \%)$ & 41 (95\%) & NS \\
\hline Local protocols for fever control & $45(88 \%)$ & $34(79 \%)$ & NS \\
\hline $\begin{array}{l}\text { Local protocols for reducing elevated blood } \\
\text { glucose levels }\end{array}$ & $44(86 \%)$ & $37(86 \%)$ & NS \\
\hline Local protocols for swallow dysfunction & $45(88 \%)$ & $41(95 \%)$ & NS \\
\hline \multicolumn{4}{|l|}{$\begin{array}{l}\text { Protocol for referral to the following health } \\
\text { professionals: }\end{array}$} \\
\hline Physiotherapist & $34(67 \%)$ & $36(84 \%)$ & NS \\
\hline Speech pathologist & $16(31 \%)$ & 39 (91\%) & $<0.001$ \\
\hline Occupational therapist & $17(33 \%)$ & $36(84 \%)$ & $<0.001$ \\
\hline Dietician & $24(47 \%)$ & $37(86 \%)$ & $<0.001$ \\
\hline Psychologist & $22(43 \%)$ & $22(51 \%)$ & NS \\
\hline Psychiatrist & $9(18 \%)$ & $21(49 \%)$ & 0.002 \\
\hline Social worker & $6(12 \%)$ & $35(81 \%)$ & $<0.001$ \\
\hline $\begin{array}{l}\text { Swallowing screen performed within } 24 \\
\text { hours for all stroke patients }\end{array}$ & $28(56 \%)$ & 34 (79\%) & 0.027 \\
\hline $\begin{array}{l}\text { Formal swallowing assessment by a speech } \\
\text { pathologist or speech and language therapist } \\
\text { performed on referral }\end{array}$ & $17(36 \%)$ & $31(72 \%)$ & 0.001 \\
\hline $\begin{array}{l}\text { Discharge summary sent to the General } \\
\text { Practitioner }\end{array}$ & $16(36 \%)$ & 41 (95\%) & $<0.001$ \\
\hline $\begin{array}{l}\text { Routine outpatient clinic follow-up review } \\
\text { for all stroke patients }\end{array}$ & $41(87 \%)$ & $37(86 \%)$ & NS \\
\hline Early supported discharge & $29(60 \%)$ & $33(77 \%)$ & NS \\
\hline
\end{tabular}


home-based rehabilitation and assessment

Access to on-going rehabilitation at the hospital or at another hospital

$44(92 \%) \quad 41(95 \%) \quad$ NS

Home nursing service available for stroke patients

$11(23 \%) \quad 26(63 \%) \quad<0.001$

Access to palliative care services

$17(37 \%) \quad 42(98 \%) \quad<0.001$

Nursing staff attend continuing education on stroke management

$42(86 \%) \quad 39(91 \%) \quad$ NS

Quality improvement activities in the last 2

years for the stroke team

$36(75 \%) \quad 40(93 \%) \quad 0.007$

Data are presented in number and percentage or median and interquartile range (IQR) ED: emergency department, AIS: acute ischemic stroke, ICH: intracerebral hemorrhage, MIC: middle income countries, HIC: high income countries, rtPA: recombinant tissue plasminogen activator, NS: not significant. P value from Chi squared, Fisher or MannWithney test as appropriate. 
Table 3: Human resources in stroke care by country income

Onsite specialist physicians responsible for
stroke patients
Number of patients to one staff member in
ED:

Nurse

Health assistant

$4(3-7)$

NS

6 (4-8)

NS

Number of patients to one staff member in the stroke care unit:

\begin{tabular}{|c|c|c|c|}
\hline Nurse & $6(4-9)$ & $6(4-8)$ & NS \\
\hline Health assistant & $5.5(2-10)$ & $7(6-8)$ & NS \\
\hline $\begin{array}{l}\text { Medical staff from the stroke service } \\
\text { available in ED }\end{array}$ & $34(68 \%)$ & $36(88 \%)$ & 0.044 \\
\hline \multicolumn{4}{|l|}{$\begin{array}{l}\text { Medical officers who attend a typical ward in } \\
\text { the stroke care unit: }\end{array}$} \\
\hline Consultants - weekday & $3(1-10)$ & $1(1-2)$ & 0.012 \\
\hline Consultants - weekend & $1(0-5)$ & $1(0-1)$ & 0.048 \\
\hline Fellow/advanced trainees - weekday & $3(1-5)$ & $1(0-1)$ & $<0.001$ \\
\hline Fellow/advanced trainees - weekend & $1(0-1)$ & $0(0-0)$ & $<0.001$ \\
\hline Registrars - weekday & $4(2-6)$ & $1(1-1)$ & $<0.001$ \\
\hline Registrars - weekend & $1(1-2)$ & $0(0-1)$ & $<0.001$ \\
\hline Junior medical officers - weekday & $4(2-7)$ & $2(1-3)$ & $<0.001$ \\
\hline Junior medical officers - weekend & $2(1-3)$ & $0(0-1)$ & $<0.001$ \\
\hline $\begin{array}{l}\text { Special doctor rostered after hours to attend } \\
\text { specifically to stroke patients who require } \\
\text { admission to hospital }\end{array}$ & $44(92 \%)$ & $26(61 \%)$ & $<0.001$ \\
\hline Dedicated stroke team & $42(82 \%)$ & $41(95 \%)$ & NS \\
\hline $\begin{array}{l}\text { Multidisciplinary stroke team meetings held } \\
\text { at least weekly }\end{array}$ & $24(48 \%)$ & 38 (93\%) & $<0.001$ \\
\hline $\begin{array}{l}\text { Stroke unit team provide medical care or } \\
\text { advice for patients not in the stroke care unit }\end{array}$ & 36 (78\%) & 42 (100\%) & 0.001 \\
\hline
\end{tabular}



Figure: Percentage of hospitals having different health professionals involved in the management of stroke patients, by country income

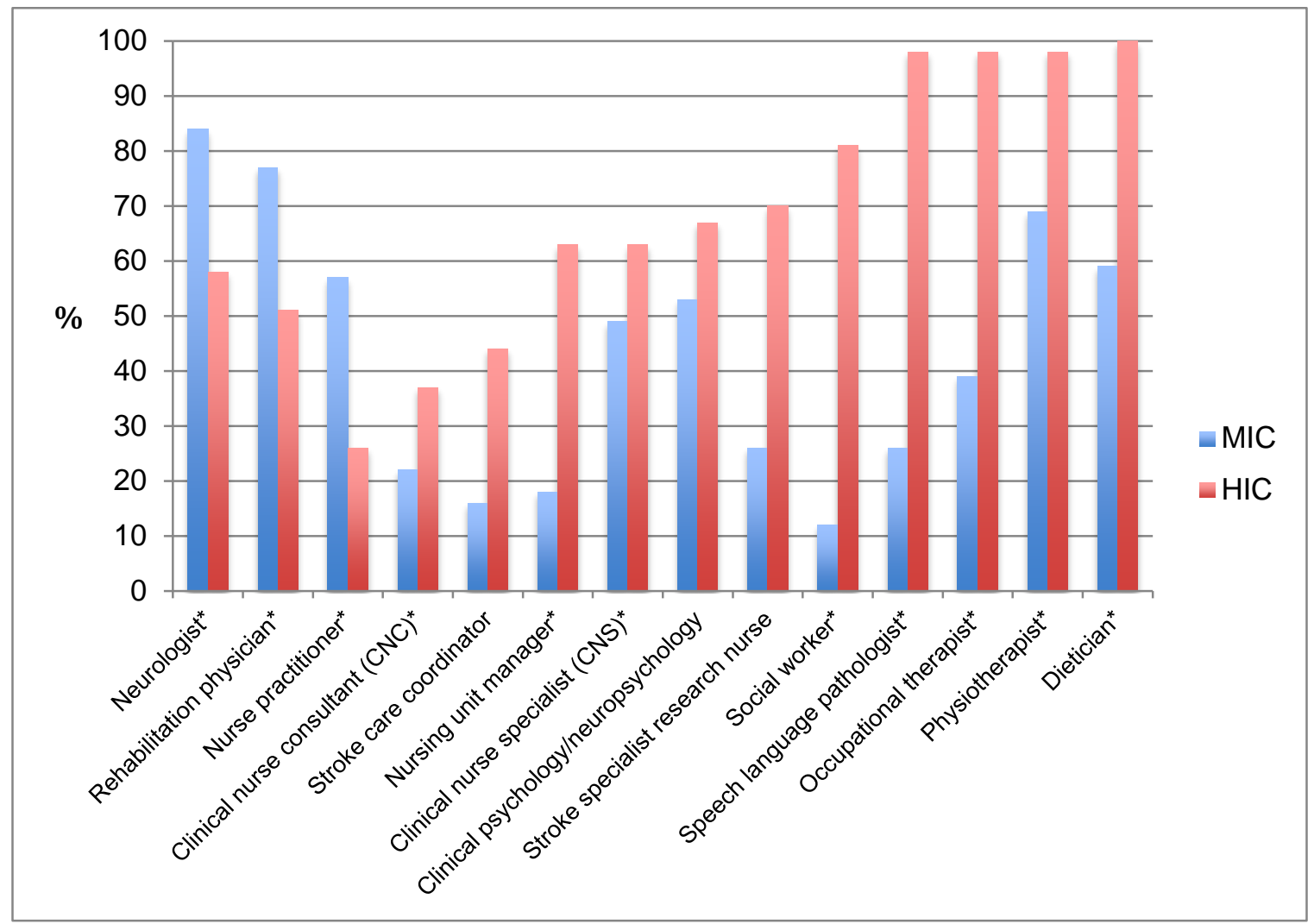

* indicates a statistically significant difference, with $\mathrm{P}$ value $<0.05$

MIC: middle-income countries, HIC: high-income countries 